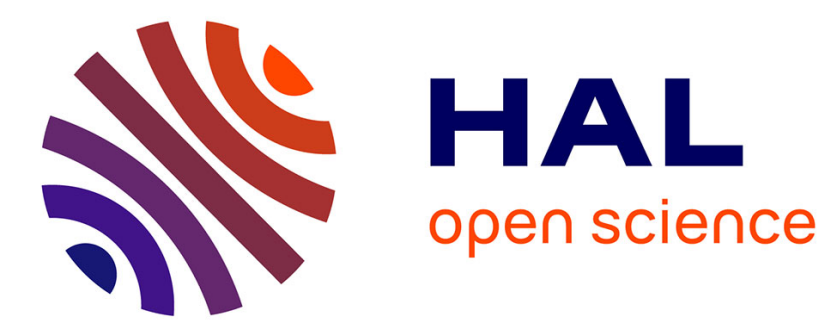

\title{
On some conjectures of P. Barry
}

J.-P. Allouche, Guo-Niu Han, J. Shallit

\section{To cite this version:}

J.-P. Allouche, Guo-Niu Han, J. Shallit. On some conjectures of P. Barry. Journal of Number Theory, 2021, 228, pp.108-132. 10.1016/j.jnt.2021.03.015 . hal-03379172

\section{HAL Id: hal-03379172 \\ https://hal.science/hal-03379172}

Submitted on 14 Oct 2021

HAL is a multi-disciplinary open access archive for the deposit and dissemination of scientific research documents, whether they are published or not. The documents may come from teaching and research institutions in France or abroad, or from public or private research centers.
L'archive ouverte pluridisciplinaire HAL, est destinée au dépôt et à la diffusion de documents scientifiques de niveau recherche, publiés ou non, émanant des établissements d'enseignement et de recherche français ou étrangers, des laboratoires publics ou privés. 


\title{
On some conjectures of P. Barry
}

\author{
J.-P. Allouche \\ G.-N. Han \\ CNRS, IMJ-PRG \\ CNRS, IRMA \\ Sorbonne Université \\ 4 Place Jussieu \\ F-75252 Paris Cedex 05 France \\ jean-paul.allouche@imj-prg.fr \\ Université de Strasbourg \\ 7 rue René Descartes \\ F-67084 Strasbourg France \\ guoniu.han@unistra.fr
}

\author{
J. Shallit \\ School of Computer Science \\ University of Waterloo \\ Waterloo, ON N2L 3G1 \\ Canada \\ shallit@uwaterloo.ca
}

January 16, 2021

\begin{abstract}
We prove a number of conjectures recently stated by P. Barry, related to the paperfolding sequence and the Rueppel sequence. Furthermore, we study the regularity of sequences involved in the paper, and prove that for all $q \geq 2$, the sequence consisting of the positive integers whose odd part is of the form $4 k+1$ is not $q$-regular. Finally we establish the 2-regularity of two sequences of Hankel determinants.
\end{abstract}

AMS 2010 Classifications: Primary 11B85, 68R15 Secondary 11C20, 05A15, 15A15.

Keywords: Hankel determinant, Rueppel sequence, paperfolding sequence, automatic sequence, regular sequence.

\section{Introduction}

In his recent paper [5], P. Barry studied a number of integer sequences - in particular, the (regular) paperfolding sequence and the Rueppel sequence. Recall that the sequence $\left(j_{n}\right)_{n \geq 0}$ is defined by $j_{0}=0$, and, for $n>0$, by the Jacobi-Kronecker symbol $j_{n}=\left(\frac{-1}{n}\right)$. It is sequence 
A034947 in the OEIS [15], and is, up to the first term, the \pm 1 paperfolding sequence, as noted by J. Sondow and recalled in [5]. It can thus be also defined by the relations

$$
j_{0}=0, j_{2 k}=j_{k} \text { for all } k \geq 0 \text {, and } j_{2 k+1}=(-1)^{k} \text { for all } k \geq 0 \text {. }
$$

or the generating function

$$
\sum_{n \geq 0} j_{n} x^{n}=\sum_{k \geq 0} \frac{x^{2^{k}}}{1+x^{2^{k+1}}} .
$$

The Rueppel sequence $\left(r_{n}\right)_{n \geq 0}=(1,1,0,1,0,0,0,1, \ldots)$ is the characteristic sequence of the set $\left\{2^{n}-1: n \geq 0\right\}$. Alternatively, it is defined by the generating function

$$
r(x)=\sum_{n \geq 0} r_{n} x^{n}=\sum_{n \geq 0} x^{2^{n}-1}=1+x+x^{3}+x^{7}+x^{15}+\cdots
$$

One reason that $\mathrm{P}$. Barry studied Rueppel sequence is its relation with the famous Catalan sequence $[5,6,9]$

$$
r_{n} \equiv C_{n}=\frac{1}{n+1}\left(\begin{array}{c}
2 n \\
n
\end{array}\right) \quad(\bmod 2) .
$$

We identify a sequence $\mathbf{a}=\left(a_{0}, a_{1}, a_{2}, \ldots\right)$ and its generating function $f=f(x)=$ $a_{0}+a_{1} x+a_{2} x^{2}+\cdots$. Usually, $a_{0}=1$. For each $n \geq 1$ the Hankel determinant of the series $f$ (or of the sequence a) is defined by

$$
H_{n}(f):=\left|\begin{array}{cccc}
a_{0} & a_{1} & \ldots & a_{n-1} \\
a_{1} & a_{2} & \ldots & a_{n} \\
\vdots & \vdots & \ddots & \vdots \\
a_{n-1} & a_{n} & \ldots & a_{2 n-2}
\end{array}\right| .
$$

We let $H_{0}(f)=1$. The sequence of the Hankel determinants of $f$ is defined as follows:

$$
H(f):=\left(H_{0}(f), H_{1}(f), H_{2}(f), H_{3}(f), \ldots\right) .
$$

In [5] Barry states several conjectures related to the (regular) paperfolding sequence and the Hankel determinants of the modified Rueppel sequences. In Section 2 we prove the first three conjectures, related to the paperfolding sequence. Next, we prove that for all $q \geq 2$, the sequence consisting of the positive integers whose odd part is of the form $4 k+1$ is not $q$-regular. We suggest some further questions in Section 3. In Sections 4-6, we study the Hankel determinants of $1-x r(x), 1+x r(x)$, and $r(x) /(r(x)-x)$, and prove Barry's Conjectures 6, 7, 8, 9, 10,11, and 16, respectively. Also, we establish the 2-regularity of two sequences of Hankel determinants. 


\section{On the first three conjectures of Barry}

One important sequence $\left(s_{n}\right)_{n \geq 0}$ in [5] is the sequence A088748 in the OEIS [15] defined by

$$
s_{n}=1+\sum_{0 \leq k \leq n} j_{k}
$$

or by the generating function

$$
\sum_{n \geq 0} s_{n} x^{n}=\frac{1}{1-x}\left(1+\sum_{k \geq 0} \frac{x^{2^{k}}}{1+x^{2^{k+1}}}\right) .
$$

Proposition 1. The sequence $\left(s_{n}\right)_{n \geq 0}$ satisfies, for all $n \geq 0$, the relations

$$
\begin{gathered}
s_{2 n}=s_{n}+ \begin{cases}0, & \text { if } n \text { is even; } \\
1, & \text { if } n \text { is odd }\end{cases} \\
s_{2 n+1}=s_{n}+ \begin{cases}1, & \text { if } n \text { is even; } \\
0, & \text { if } n \text { is odd }\end{cases}
\end{gathered}
$$

Proof. We write

$$
s_{2 n}=1+\sum_{0 \leq k \leq 2 n} j_{k}=1+\sum_{0 \leq m \leq n} j_{2 m}+\sum_{0 \leq m \leq n-1} j_{2 m+1}=1+\sum_{0 \leq m \leq n} j_{m}+\sum_{0 \leq m \leq n-1}(-1)^{m} .
$$

Hence

$$
s_{2 n}=s_{n}+\sum_{0 \leq m \leq n-1}(-1)^{m}=s_{n}+ \begin{cases}0, & \text { if } n \text { is even; } \\ 1, & \text { if } n \text { is odd }\end{cases}
$$

This implies

$$
s_{2 n+1}=s_{2 n}+j_{2 n+1}=s_{2 n}+(-1)^{n}=s_{n}+ \begin{cases}1, & \text { if } n \text { is even } \\ 0, & \text { if } n \text { is odd }\end{cases}
$$

Theorem 2. Barry's Conjecture 1 is true: the locations of the occurrences of $m$ in the sequence $\left(s_{n}\right)_{n \geq 0}$ are given by those numbers whose base-2 representation has exactly $m-1$ runs. Furthermore the values of $m$ occurring in $\left\{s_{0}, s_{1}, \ldots, s_{2^{N}-1}\right\}$ are $1,2, \ldots, N+1$.

Proof. To prove that the statement of the theorem holds for all indices $n$ of $\left(s_{n}\right)_{n \geq 0}$, we prove by induction on $N$ that the property holds for all $m$ occurring at indices $n \in\left[0,2^{N}-1\right]$ of $\left(s_{n}\right)_{n \geq 0}$.

The claim holds for $N=0$, since the only index to consider is then $n=0$, and $s_{0}=1$, while the binary expansion of 0 is empty, and hence has no runs.

Suppose that the desired property holds for all $n \in\left[0,2^{N}-1\right]$. Every number $n \in$ $\left[0,2^{N+1}-1\right]$ can be written $n=2 a+r$ where $r \in\{0,1\}$. Thus $a$ is necessarily in $\left[0,2^{N}-1\right]$. There are four cases. We have, using Proposition 1: 
- if $r=0$ and $a$ even, say $a=2 b$, then $n=4 b$. Thus $s_{n}=s_{4 b}=s_{2 b}=s_{a}$. Since $n=4 b$ and $a=2 b$ have the same number of runs, the property holds for $n$.

- if $r=0$ and $a$ odd, say $a=2 b+1$, then $n=4 b+2$. Thus $s_{n}=s_{4 b+1}=s_{2 b}+1$. Since $n=4 b+2$ clearly has one more run then $a=2 b+1$, the property holds for $n$.

- if $r=1$ and $a$ even, say $a=2 b$, then $n=4 b+1$. Thus $s_{n}=s_{4 b+1}=s_{2 b}+1$. Since $n=4 b+1$ has one more run than $a=2 b$, the property holds for $n$.

- if $r=1$ and $a$ odd, say $a=2 b+1$, then $n=4 b+3$. Thus $s_{n}=s_{4 b+3}=s_{2 b+1}$. Since $n=4 b+3$ and $a=2 b+1$ have the same number of runs, the property holds for $n$.

This completes the proof.

Remark 3. An alternative statement of Theorem 2 is that the sum $\sum_{0<k<n} j_{k}$ is equal to the number of runs in the binary expansion of $n$ : this was noted by G. W. Adamson in a 2008 comment on A005811 in the OEIS [15].

Now we address two more conjectures of Barry in [5]. First we prove a general statement.

Proposition 4. Let $\left(c_{n}\right)_{n \geq 0}$ be an increasing sequence of integers. Let $\left(\lambda_{n}\right)_{n \geq 0}$ be the characteristic function of the set $\left\{c_{0}, c_{1}, \ldots, c_{n}, \ldots\right\}$. Then

$$
\forall n \geq 0, \quad c_{n}-\sum_{0 \leq k \leq c_{n}}(-1)^{\lambda_{k}}=2 n+1
$$

Proof. First we note the equivalence

$$
c_{n}=r \Longleftrightarrow\left(\lambda_{r}=1 \text { and } \sum_{0 \leq k \leq r} \lambda_{k}=n+1\right) .
$$

But $(-1)^{\lambda_{k}}=1-2 \lambda_{k}$. Hence if $c_{n}=r$, then $\sum_{0 \leq k \leq r} \lambda_{k}=n+1$. Thus

$\sum_{0 \leq k \leq c_{n}}(-1)^{\lambda_{k}}=\sum_{0 \leq k \leq r}(-1)^{\lambda_{k}}=\sum_{0 \leq k \leq r}\left(1-2 \lambda_{k}\right)=r+1-2 \sum_{0 \leq k \leq r} \lambda_{k}=r+1-(2 n+2)=c_{n}-2 n-1$.

Remark 5. Proposition 4 is related to the problem of computing the index $n$ of the $n$th term of an increasing sequence of integers. In this direction the reader can consult [12].

Theorem 6. Barry's Conjecture 2 is true. Namely, define $a_{0}=0$, and let $\left(a_{n}\right)_{n \geq 1}$ denote the increasing sequence of positive integers whose odd part is of the form $4 k+1$. Then we have $a_{n}+s_{a_{n}}=2 n+1$. 


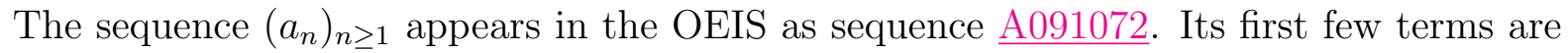
listed below:

$$
\left(a_{n}\right)_{n \geq 1}=(1,2,4,5,8,9,10,13,16,17,18,20,21,25, \ldots)
$$

Proof. We note that the recursive definition of $j_{n}$ implies that the sequence $\left(a_{n}\right)_{n \geq 0}$ is exactly the sequence of integers consisting of 0 and the integers $m \geq 1$ such that $j_{m}=1$. In other words, the characteristic function of the set $\left\{a_{0}, a_{1}, a_{2}, \ldots, a_{n}, \ldots\right\}$ is the sequence $\left(\lambda_{n}\right)_{n \geq 0}$ with $\lambda_{0}=1$ and $\lambda_{n}=\left(1+j_{n}\right) / 2$ for $n \geq 1$. So $(-1)^{\lambda_{0}}=-1$ and $(-1)^{\lambda_{k}}=-j_{k}$ for $k \geq 1$. Thus Proposition 4 above with $\left(c_{n}\right)_{n \geq 0}=\left(a_{n}\right)_{n \geq 0}$ yields

$$
a_{n}+s_{a_{n}}=a_{n}+1+\sum_{1 \leq k \leq a_{n}} j_{k}=a_{n}-\sum_{0 \leq k \leq a_{n}}(-1)^{\lambda_{k}}=2 n+1 .
$$

Theorem 7. Barry's Conjecture 3 is true. Let $\left(b_{n}\right)_{n \geq 0}$ denote the increasing sequence of integers whose odd part is of the form $4 k+3$. Then we have $b_{n}-s_{b_{n}}=2 n+1$.

The sequence $\left(b_{n}\right)_{n \geq 1}$ appeared in the OEIS as sequence $\underline{A 091067}$. Its first few terms are listed below:

$$
\left(b_{n}\right)_{n \geq 1}=(3,6,7,11,12,14,15,19,22,23,24,27,28,30,31, \ldots)
$$

Proof. The integers in the sequence $\left(b_{n}\right)_{n \geq 0}$ are exactly the integers $m \geq 0$ for which $j_{m}=-1$. Hence the characteristic function of $\left(c_{n}\right)_{n \geq 1}$ is $\left(\mu_{n}\right)_{n \geq 0}$ with $\mu_{0}=0$ and $\mu_{n}=\left(1-j_{n}\right) / 2$ for $n \geq 1$. So $(-1)^{\mu_{0}}=1$ and $(-1)^{\mu_{k}}=j_{k}$ for $k \geq 1$. Now we apply Proposition 4 with $\left(c_{n}\right)_{n \geq 0}=$ $\left(b_{n}\right)_{n \geq 0}$ to get

$$
b_{n}-s_{b_{n}}=b_{n}-1-\sum_{1 \leq k \leq b_{n}} j_{k}=b_{n}-\sum_{0 \leq k \leq a_{n}}(-1)^{\mu_{k}}=2 n+1 .
$$

We conclude this section with a table giving the first few terms of the sequences we have discussed.

\begin{tabular}{|c|ccccccccccccccccc}
$n$ & 0 & 1 & 2 & 3 & 4 & 5 & 6 & 7 & 8 & 9 & 10 & 11 & 12 & 13 & 14 & 15 & 16 \\
\hline$j_{n}$ & 0 & 1 & 1 & -1 & 1 & 1 & -1 & -1 & 1 & 1 & 1 & -1 & -1 & 1 & -1 & -1 & 1 \\
$s_{n}$ & 1 & 2 & 3 & 2 & 3 & 4 & 3 & 2 & 3 & 4 & 5 & 4 & 3 & 4 & 3 & 2 & 3 \\
$a_{n}$ & 0 & 1 & 2 & 4 & 5 & 8 & 9 & 10 & 13 & 16 & 17 & 18 & 20 & 21 & 25 & 26 & 29 \\
$b_{n}$ & 3 & 6 & 7 & 11 & 12 & 14 & 15 & 19 & 22 & 23 & 24 & 27 & 28 & 30 & 31 & 35 & 38
\end{tabular}




\section{A (non-)regularity property of the sequence $\left(a_{n}\right)_{n \geq 0}$}

While writing this paper, we realized that a result similar to Theorem 6 and to Theorem 7 was proved in [1], where the paperfolding sequence was replaced with a generalized ThueMorse sequence. For example, let $\left(t_{n}\right)_{n \geq 0}$ be the usual Thue-Morse sequence (see, e.g., [3]), where $t_{n}$ is the parity of the sum of the binary digits of $n$. Let $\left(u_{n}\right)_{n \geq 0}$ denote the increasing sequence of odious numbers, namely the numbers $n$ for which $t_{n}=1$, and $\left(v_{n}\right)_{n \geq 0}$ be the increasing sequence of evil numbers, namely the numbers $n$ for which $t_{n}=0$. Then (see $[1$, Corollary 1, p. 34]) we have

$$
\forall n \geq 0, u_{n}=2 n+1-t_{n}, \text { and } v_{n}=2 n+t_{n}
$$

This property can be compared with Proposition 4. Namely, for all $n \geq 0$, we have $t_{2 n}=t_{n}$ and $t_{2 n+1}=1-t_{n}$. Thus

$$
\sum_{0 \leq k \leq n}(-1)^{t_{k}}= \begin{cases}0, & \text { if } n \text { is odd } \\ (-1)^{t_{n}}, & \text { if } n \text { is even }\end{cases}
$$

Proposition 4 thus implies that (noting that for all $n$ one has $t_{u_{n}}=1$ )

$$
\forall n \geq 0,2 n+1=u_{n}-\sum_{0 \leq k \leq u_{n}}(-1)^{t_{k}}= \begin{cases}u_{n}, & \text { if } u_{n} \text { is odd } \\ u_{n}-(-1)^{t_{u_{n}}}=u_{n}+1, & \text { if } u_{n} \text { is even }\end{cases}
$$

This only says that $\left\lfloor\frac{u_{n}}{2}\right\rfloor=n$ (and similarly $\left\lfloor\frac{v_{n}}{2}\right\rfloor=n$ ), which is clearly implied by, but weaker than, the identities in (4), which state that $u_{n}=1-t_{n} \bmod 2$ and $v_{n}=t_{n} \bmod 2$.

Recall that the $r$-kernel of a sequence $\left(x_{n}\right)_{n \geq 0} \in \mathbb{Z}^{\mathbb{N}}$, for an integer $d \geq 2$, is the set of subsequences

$$
\left\{\left(x_{r^{i} n+j}\right)_{n \geq 0}, i \geq 0, j \in\left[0, r^{i}-1\right]\right\} .
$$

Also recall that a sequence $\left(x_{n}\right)_{n \geq 0}$ is called $r$-automatic if its $r$-kernel is finite, and $r$-regular if its $r$-kernel generates a $\mathbb{Z}$-module of finite type (see [4]).

What precedes implies, in particular, that the sequences $\left(u_{n}\right)_{n \geq 0}$ and $\left(v_{n}\right)_{n \geq 0}$ are 2regular, while it is well-known that the Thue-Morse sequence $\left(t_{n}\right)_{n \geq 0}$ is 2-automatic. Also note the asymptotic behavior $u_{n} \sim v_{n} \sim 2 n$, for $n \rightarrow \infty$.

On the other hand the sequence $\left(j_{n}\right)_{n \geq 0}$, being equal to the \pm 1 -paperfolding sequence up to its first term, is 2-automatic. Also, we clearly have, from Remark 3 , that $s_{n}$ is at most one plus half the number of digits of $n$. Thus, using Theorem 2, we see that $s_{n}=\mathcal{O}(\log n)$, and so $a_{n} \sim 2 n$. Hence $a_{n}=2 n+\mathcal{O}\left(\log a_{n}\right)=2 n+\mathcal{O}(\log n)$. Similarly, using Theorem 6 , we get $b_{n}=2 n+\mathcal{O}(\log n)$.

A question that now comes to mind is whether $\left(a_{n}\right)_{n \geq 0}$ and $\left(b_{n}\right)_{n \geq 0}$ are 2-regular sequences. We prove that they are not. First, we prove a proposition characterizing the integers $n$ for which $a_{n+1}-a_{n}=2$. 


\section{Proposition 8.}

(i) We have $a_{n+1}-a_{n}=2$ for some integer $n$ if and only if there exists an integer $r$ such that $n=8 r+\frac{1+s_{16 r+2}}{2}$. Then $a_{n}=16 r+2$ and such an $r$ is unique, with $r=\left\lfloor\frac{a_{n}}{16}\right\rfloor$.

(ii) Let denote $\psi(r)=8 r+\frac{1+s_{16 r+2}}{2}$, so that $a_{\psi(r)}=16 r+2$ and $r=\left\lfloor\frac{a_{\psi(r)}}{16}\right\rfloor$. The function $\psi$ is increasing for $r \geq 0$. Furthermore, for all $r, r^{\prime}$ with $r \neq r^{\prime}$ we have $\left|\psi(r)-\psi\left(r^{\prime}\right)\right| \geq 7$.

Proof.

(i) First we note that $\frac{1+s_{16 r+2}}{2}$ is always an integer: the number of runs of an even number is even, and hence $s(m)$ must be odd when $m$ is an even integer.

Now let $n$ be such that $a_{n+1}-a_{n}=2$. Write $a_{n}=4 k+j$ with $j \in[0,3]$. Let $A$ denote the set of values of the sequence $\left(a_{n}\right)_{n \geq 0}: A=\{0,1,2,4,5,8,9,10,13,16,17,18,20,21, \ldots\}$ We have that $j \neq 0$, for otherwise $a_{n}+1=4 k+1 \in A$ and $a_{n+1}=a_{n}+1$. We also have $j \neq 1$, for otherwise $a_{n}+2 \equiv 3(\bmod 4)$ does not belong to $A$, and hence cannot equal $a_{n+2}$. Since $j \neq 3$ because $4 k+3 \notin A$, we must have $a_{n}=4 k+2$. Since $4 k+2$ belongs to $A$ if and only if $2 k+1$ belongs to $A$, if and only if $k$ is even, there exists $\ell$ with $k=2 \ell$. But then $a_{n+1}=a_{n}+2=4 k+4=8 \ell+4$ belongs to $A$ : this is equivalent to saying that $2 \ell+1$ belongs to $A$, which holds if and only if $\ell$ is even, say $\ell=2 r$. Thus $a_{n}=4 k+2=16 r+2$. Theorem 6 then implies that $16 r+2+s_{16 n+2}=2 n+1$. Hence $n=8 r+\frac{1+s_{16 r+2}}{2}$.

Conversely, suppose that there exists an integer $r$ such that $n=8 r+\frac{1+s_{16 r+2}}{2}$. The number $16 n+r$ belongs to $A$, and hence there exists an integer $m$ with $a_{m}=16 r+2$. Since $a_{m}+1=16 r+3$ does not belong to $A$, and $a_{m}+2=16 r+4=4(4 r+1)$ belongs to $A$, we have that $a_{m+1}=a_{m}+2$. Hence $a_{m+1}-a_{m}=2$. To finish the proof, we claim that $m=n$. Using the first part of the proof, we have that $m=8 r+\frac{1+s_{16 r+2}}{2}$. Hence $m=n$.

(ii) To prove that $\psi$ is increasing and that $\left|\psi(r)-\psi\left(r^{\prime}\right)\right| \geq 7$, it suffices to prove the inequality $\psi(r+1)-\psi(r) \geq 7$ for all $r \geq 0$. Thus it suffices to prove that $8+$ $\frac{s_{16 r+18}-s_{16 r+2}}{2} \geq 7$, for all $r \geq 0$. This last inequality would be implied by the inequality $\left|s_{16 r+18}-s_{16 r+2}\right| \leq 2$. But the properties of $s_{n}$ given in Proposition 1 can be rewritten as follows:

$$
\left\{\begin{aligned}
s_{4 n} & =s_{2 n} \\
s_{4 n+1} & =s_{2 n}+1 \\
s_{4 n+2} & =s_{2 n+1}+1 \\
s_{4 n+3} & =s_{2 n+1} .
\end{aligned}\right.
$$

Therefore $s_{16 n+2}=s_{8 n+1}+1=s_{4 n}+2=s_{2 n}+2$. Hence

$$
s_{16 r+18}-s_{16 r+2}=s_{16(r+1)+2}-s_{16 r+2}=s_{2 r+2}-s_{2 r} .
$$


We distinguish two cases according to the parity of $r$ :

$$
\begin{gathered}
\text { If } r=2 t \text {, then } s_{2 r+2}-s_{2 r}=s_{4 t+2}-s_{4 t}=s_{2 t+1}+1-s_{2 t}=s_{r+1}-s_{r}+1 ; \\
\text { If } r=2 t+1 \text {, then } s_{2 r+2}-s_{2 r}=s_{4 t+4}-s_{4 t+2}=s_{2 t+2}-s_{2 t+1}-1=s_{r+1}-s_{r}-1 .
\end{gathered}
$$

Now it suffices to prove that $\left|s_{n+1}-s_{n}\right| \leq 1$, for all $n \geq 0$. But $s_{n}$ is equal to $1+$ the number of runs in the binary expansion of $n$ (see Remark 3 above). And it is easy to see that the absolute value of the difference of the number of runs for two consecutive integers is equal to 1 (see, e.g., the remark before Theorem 4 in [16]).

Theorem 9. For all $q \geq 2$ the sequence $\left(a_{n}\right)_{n \geq 0}$ is not $q$-regular.

Proof. If the sequence $\left(a_{n}\right)_{n \geq 0}$ were $q$-regular, its difference sequence, say $\left(d_{n}\right)_{n \geq 0}$, where $d_{n}=a_{n+1}-a_{n}$, would be $q$-regular as well. But $\left(d_{n}\right)_{n \geq 0}$ takes only finitely many values: recall that all integers congruent to 1 modulo 4 are values of $a_{n}$, so that $d_{n} \in[1,4]$. Thus, if $\left(d_{n}\right)_{n \geq 0}$ were $q$-regular, it would be $q$-automatic. The proof that the sequence $\left(d_{n}\right)_{n \geq 0}$ is not $q$-automatic is given in the next three theorems: we first prove that $\left(d_{n}\right)_{n \geq 0}$ is not 2 -automatic. Next, we prove that it is morphic. Finally, we prove that for all $q \geq 2$ it is not $q$-automatic.

Theorem 10. The sequence $\left(d_{n}\right)_{n \geq 0}$ is not 2-automatic.

Proof. Since a 2-automatic sequence $\left(x_{n}\right)_{n \geq 0}$ is characterized by the fact that its 2-kernel (i.e., the set of subsequences $\left.\left\{\left(x_{2^{k} n+j}\right)_{n \geq 0}, k \geq 0, j \in\left[0,2^{k}-1\right]\right\}\right)$ is finite, it suffices to prove that the subsequences $\left(u_{\alpha}(n)\right)_{n \geq 0}$ and $\left(u_{\beta}(n)\right)_{n \geq 0}$ are distinct, where $u_{\alpha}(n):=d\left(2^{2^{\alpha}} n+2^{\alpha}\right)$ and $u_{\beta}(n)=d\left(2^{2^{\beta}} n+2^{\beta}\right)$ with $\alpha<\beta$ and $\alpha$ large enough. First note that

$$
t=\underbrace{1010 \cdots 10}_{j \text { blocks } 10} \text { (in base } 2) \Longrightarrow t=\frac{2}{3}\left(2^{2 j}-1\right) \text { and } s(t)=2 j+1 \text {. }
$$

Now take $j=2^{\alpha}-2$ and $t=\frac{2}{3}\left(2^{2^{\alpha+1}-4}-1\right)$, so that $s(t)=2^{\alpha+1}-3$. Define $r=2^{2^{\alpha}-3} t$. Then $s(16 r+2)=s\left(2^{2^{\alpha}+1} t+2\right)=2+s(2 t)=2+s(t)$ (recall that $t$ is even). So $s(16 r+2)=$ $2+2^{\alpha+1}-3=2^{\alpha+1}-1$. Hence $\psi(r)=8 r+2^{\alpha}=2^{2^{\alpha}} t+2^{\alpha}$. We deduce, using Proposition 8 , that $u_{\alpha}(t)=2$.

Now we prove that $u_{\beta}(t) \neq 2$. Define $r^{\prime}=2^{2^{\beta}-3} t+2^{\beta-3}-2^{\alpha-3}$. Then

$$
\begin{aligned}
s\left(16 r^{\prime}+2\right) & =s\left(2^{2^{\beta}+1} t+2^{\beta+1}-2^{\alpha+1}+2\right) \\
& =s(2 t)+s\left(2^{\beta+1}-2^{\alpha+1}+2\right) \\
& =s(2 t)+4=s(t)+4=2^{\alpha+1}+1 .
\end{aligned}
$$

Hence $\psi\left(r^{\prime}\right)=8 r^{\prime}+\frac{1+s\left(16 r^{\prime}+2\right)}{2}=2^{2^{\beta}} t+2^{\beta}+1$. Thus $\psi\left(r^{\prime}\right)-\left(2^{2^{\beta}} t+2^{\beta}\right)=1$. Since $\left|\psi\left(r^{\prime \prime}\right)-\psi\left(r^{\prime}\right)\right| \geq 7$ for all $r^{\prime \prime} \neq r^{\prime}$ (Proposition 8), the integer $\left(2^{2^{\beta}} t+2^{\beta}\right)$ cannot be equal to some $\psi\left(r^{\prime \prime}\right)$. Hence $u_{\beta}(t)=a\left(2^{2^{\beta}} t+2^{\beta}\right) \neq 2$ (again from Proposition 8). Hence $u_{\beta}(t) \neq u_{\alpha}(t)$, so that the sequences $u_{\beta}$ and $u_{\alpha}$ are distinct. A similar proof gives that $\left(b_{n}\right)_{n \geq 0}$ is not 2regular. 
The next theorem proves that the sequence $\left(d_{n}\right)_{n \geq 0}$ is morphic. For more about morphic sequences, see, e.g., [4].

Theorem 11. The sequence $\mathbf{d}=\left(d_{n}\right)_{n \geq 0}$ is morphic. More precisely, let $\mathbf{d}^{\prime}=\left(d_{n+1}\right)_{n \geq 0}$. If we define the morphisms $f$ on $\{0,1,2,3\}^{*}$ and $g$ from $\{0,1,2,3\}^{*}$ to $\{1,2,3,4\}^{*}$ as follows:

$$
f(0)=01, f(1)=21, f(2)=03, f(3)=23,
$$

and

$$
g(0)=121, g(1)=31, g(2)=13, g(3)=4 .
$$

Then $\mathbf{d}^{\prime}=g\left(f^{\infty}(0)\right)$. That is, $\mathbf{d}^{\prime}=\lim _{n \rightarrow \infty} g\left(f^{(n)}(0)\right.$.

Proof. We have that $\mathbf{d}$ is morphic if and only if $\mathbf{d}^{\prime}$ is. The characteristic sequence of $\left(a_{n}\right)_{n \geq 0}$ is the paperfolding sequence $\left(p_{n}\right)_{n \geq 0}=\left(j_{n+1}\right)_{n \geq 0}$ (with the notation above). We recall that the paperfolding sequence can be defined using "perturbed symmetry" as follows: let $X_{k}=p_{0} p_{1} \cdots p_{k}$ be its prefix of length $k+1$. Then $X_{0}=1$ and for all $k \geq 0, X_{k+1}=X_{k} 1 \overline{X_{k}^{R}}$, where $W^{R}$ is the word obtained from $W$ by writing it backwards, and $\bar{W}$ is the word obtained from $W$ by replacing 0 's with 1's and 1's with 0's (see, e.g., [13] or [14]). Now let us define $U_{k}$ (resp., $V_{k}$ ) to be the word of distances between consecutive 1's (resp., consecutive 0's) in $X_{k}$. For example

$$
\begin{array}{lll}
X_{0}=1 & U_{0}=\epsilon & V_{0}=\epsilon \\
X_{1}=110 & U_{1}=0 & V_{1}=\epsilon \\
X_{2}=1101100 & U_{2}=010 & V_{2}=20 \\
X_{3}=110110011100100 & U_{3}=0102002 & V_{3}=203010
\end{array}
$$

The perturbed symmetry definition of the $X_{k}$ 's, and the fact that, for $k \geq 2, X_{k}$ begins with 11 and ends with 00 , show that

$$
U_{k+1}=U_{k} 20 V_{k}^{R} \text { and } V_{k+1}=V_{k} 3 U_{k}^{R} .
$$

The sequence of words $\left(U_{k}\right)_{k}$ (resp., $\left(V_{k}\right)_{k}$ ) clearly converges to an infinite sequence $U$ (resp., $V$ ) with values in $\{0,1,2,3\}$. It is straightforward to see that the difference between the indexes of the 1's (resp., the 0 's) in the sequence $\left(p_{n}\right)_{n \geq 0}$ are obtained by adding 1 to the terms of the sequence $U$ (resp., the sequence $W$ ). Thus, the difference of indexes of consecutive 1's (resp., consecutive 0's) in $\left(p_{n}\right)_{n \geq 0}$ (which is the difference sequence of $\left.\left(a_{n+1}\right)_{n \geq 0}\right)$ is given by the sequence $A$ (resp., $B$ ) on $\{1,2,3,4\}$ which is the limit of the sequence of words $\left(A_{k}\right)_{k}$ (resp., $\left(B_{k}\right)_{k}$ ) defined, for $k \geq 0$, as follows:

$$
A_{0}=121, B_{0}=31 \text {, and, for all } k \geq 0, A_{k+1}=A_{k} 31 B_{k}^{R} \text { and } B_{k+1}=B_{k} 4 A_{k}^{R} .
$$

If we prove that, for all $k \geq 2$, one has

$$
g\left(f^{(k)}(0)\right)=A_{k}
$$


we obtain, by letting $k$ tend to infinity, that $A=g\left(f^{\infty}(0)\right)$. To obtain (5) we prove by induction on $k \geq 1$ that

$$
A_{k}=g\left(f^{(k)}(0)\right) B_{k-1}^{R}, \quad g\left(f^{(k)}(1)\right)=B_{k-1}^{R} 31, \quad g\left(f^{(k)}(2)\right)=A_{k-1} \quad 4, \quad g\left(f^{(k)}(3)\right)=B_{k-1}^{R} 4 .
$$

First we check (6) for $k=1$ :

$$
\begin{aligned}
& g(f(0)) B_{0}^{R}=g(01) 13=g(0) g(1) 13=1213113=A_{0} 31 B_{0}^{R}=A_{1} \\
& g(f(1))=g(21)=1331=B_{0}^{R} 31 \\
& g(f(2))=g(03)=1214=A_{0} 4 \\
& g(f(3))=g(23)=134=B_{0}^{R} 4 .
\end{aligned}
$$

Now suppose that (6) holds for $k \geq 1$. Then

$$
\begin{aligned}
& g\left(f^{(k+1)}(0)\right) B_{k}^{R}=g\left(f^{(k)}(f(0)) B_{k}^{R}=g\left(f^{(k)}(01)\right) B_{k}^{R}=g\left(f^{(k)}(0)\right) g\left(f^{(k)}(1)\right) B_{k}^{R}\right. \\
& =g\left(f^{(k)}(0)\right) B_{k-1}^{R} 31 B_{k}^{R}=A_{k} 31 B_{k}^{R}=A_{k+1} ; \\
& g\left(f^{(k+1)}(1)\right)=g\left(f^{(k)}(f(1))=g\left(f^{(k)}(21)\right)=g\left(f^{(k)}(2)\right) g\left(f^{(k)}(1)\right)=A_{k-1} 4 B_{k-1}^{R} 31\right. \\
& =\left(B_{k-1} 4 A_{k-1}^{R}\right)^{R} 31=B_{k}^{R} 31 ; \\
& g\left(f^{(k+1)}(2)\right)=g\left(f^{(k)}(f(2))=g\left(f^{(k)}(03)\right)=g\left(f^{(k)}(0)\right) g\left(f^{(k)}(3)\right)=g\left(f^{(k)}(0)\right) B_{k-1}^{R} 4\right. \\
& =A_{k} 4 \text {; } \\
& g\left(f^{(k+1)}(3)\right)=g\left(f^{(k)}(f(3))=g\left(f^{(k)}(23)\right)=g\left(f^{(k)}(2)\right) g\left(f^{(k)}(3)\right)=g\left(f^{(k)}(2)\right) B_{k-1}^{R} 4\right. \\
& =A_{k-1} 4 B_{k-1}^{R} 4=\left(B_{k-1} 4 A_{k-1}^{R}\right)^{R} 4=B_{k}^{R} 4 \text {. }
\end{aligned}
$$

Remark 12. What we have proved in Theorem 11 above is that the sequence $\mathbf{d}^{\prime}$ satisfies $\mathbf{d}^{\prime}=g\left(f^{\infty}(0)\right)$. This is not exactly the definition of a morphic sequence, because $g$ is not a coding, i.e., a pointwise map, but a morphism. This is known to be equivalent to saying that $\mathbf{d}^{\prime}$ is morphic (see, e.g., [4]). Note that it might be much easier to discover and prove that a sequence $\mathbf{u}$ is equal to, say, $\beta\left(\alpha^{\infty}(0)\right)$ where $\beta$ and $\alpha$ are two morphisms, than to exhibit a morphism $\alpha_{1}$ and a coding $\varphi$ such that $\mathbf{u}=\varphi\left(\alpha_{1}^{\infty}(0)\right)$. In the first construction the morphism $\beta$ is called a decoration of the fixed point $\alpha^{\infty}(0)$ in [8].

Our last theorem proves that for all $q \geq 2$, the sequence $\left(d_{n}\right)_{n \geq 0}$ is not $q$-automatic, thus completing the proof of Theorem 9.

Theorem 13. For all $q \geq 2$, the sequence $\left(d_{n}\right)_{n \geq 0}$ is not q-automatic.

Proof. We use a deep result of Durand [10], which gave a large generalization of Cobham's theorem. Namely, from [10, Corollary 6], the sequence $\mathbf{d}^{\prime}$ is $2^{k}$-substitutive for some integer $k \geq 1$, since $f$ is a 2 -uniform morphism. So, if it were $q$-automatic for some $q \geq 2$, then either $q$ would be a power of 2 , or the sequence would be ultimately periodic [10, Theorem 1 ]. But both possibilities are ruled out by the fact that $\mathbf{d}^{\prime}$ is not 2-automatic from Theorem 10 , and hence is neither $2^{\ell}$-automatic for $\ell \geq 1$, nor ultimately periodic. 
Remark 14. A similar approach proves that the sequence $\left(b_{n}\right)_{n \geq 0}$ is not 2-regular.

Remark 15. It was already known that a sequence whose characteristic function is automatic is not necessarily regular. For example, Cateland [7] studied the expansions of integers in base $q$ with digits in $\{d, d+1, \ldots, d+q-1\}$, for some $d \in[2-q, 0]$. Using his results about integers that miss some $\operatorname{digit}(\mathrm{s})[7$, p. 90-105], one has the following:

- Let $\{0,1,3,4,9,10,12,13, \ldots\}$ be the increasing sequence of integers whose base-3 representation contains no 2 (sequence $\underline{\mathrm{A} 005836}$ in [15]). Then the characteristic function of the values of this sequence is 3-automatic, while the sequence itself is 2-regular, satisfying $z_{2 n}=3 z_{n}$ and $z_{2 n+1}=3 z_{n}+1$.

- Let $2,8,26, \ldots$ be the increasing sequence of integers whose base-3 expansion has all digits equal to 2 (this is the increasing sequence $\left.\left(3^{n}-1\right)_{n \geq 0}\right)$. The characteristic function of this sequence is 3-automatic, while the sequence itself is not $r$-regular for all $r \geq 2$ (note that it is the intersection of two 2-regular sequences).

What precedes leads to a general question.

Question 16. Let $\left(c_{n}\right)_{n \geq 0}$ be an increasing sequence of integers. Let $\left(\lambda_{n}\right)_{n \geq 0}$ be the characteristic sequence of the set $\left\{c_{0}, c_{1}, c_{2}, \ldots\right\}$. Our first question, perhaps somewhat vague and general, is what kind of conditions should sequence $\left(\lambda_{n}\right)$ satisfy in order to obtain a closed form or an asymptotic formula for $\left(c_{n}\right)$ ? In particular, in light of what precedes, if $\left(\lambda_{n}\right)_{n \geq 0}$ has some sort of regularity, does $\left(c_{n}\right)_{n \geq 0}$ inherit a "similar" regularity? For example, if $\left(\lambda_{n}\right)_{n \geq 0}$ is a q-automatic sequence, when is it true that the sequence $\left(c_{n}\right)_{n \geq 0}$ is $\ell$-regular for some $\ell \geq 2$ (where, possibly, $\ell \neq q$ )?

\section{The Hankel determinants of $1-x r(x)$}

Let

$$
B(x)=1-x r(x)=1-\sum_{k \geq 0} x^{2^{k}}=1-x-x^{2}-x^{4}-x^{8}-x^{16}-\cdots
$$

The first terms of the Hankel determinants $B(x)$ are

$$
H(B(x))=(1,1,-2,3,2,-3,4,3,2,-3,4,-5,-4,-3,4,3,2, \ldots)
$$

Consider the sequence $\left(-r_{1},-r_{2},-r_{3}, \ldots\right)$ obtained from $B(x)$ by shifting two times, i.e.,

$$
T(x)=\frac{B(x)-(1-x)}{x^{2}}=\frac{1-r(x)}{x} .
$$

It is also the negative shifted Rueppel sequence. Let $g_{n}=H_{n}(T(x))$. We establish the following characterization of $g_{n}$. 
Lemma 17. We have $g_{0}=1, g_{1}=-1$, and

$$
g_{n}=(-1)^{n+1} g_{2^{k+1}-n-1},
$$

where $2^{k}<n+1 \leq 2^{k+1}$.

Proof. First, Proposition 4 in [5] implies that $g_{0}=1$, and

$$
g_{2 n}=(-1)^{n(n+1) / 2} g_{n}, \quad g_{2 n+1}=(-1)^{(n+1)(n+2) / 2} g_{n} .
$$

Next, we prove (8) by induction on $n$ by using (9). We can verify (8) is true for $n=2,3$. Now, suppose that (8) is true for $n \leq 2 m-1$ (with $m \geq 2$ ). We consider two cases.

(i) The case $n$ even, $n=2 m$ : we need to prove that

$$
g_{2 m}=-g_{2^{k+1}-2 m-1}, \quad \text { with } \quad 2^{k}<2 m+1 \leq 2^{k+1} .
$$

Since $2^{k}<2 m+1 \leq 2^{k+1}$ is equivalent to $2^{k}<2 m+2 \leq 2^{k+1}$ or $2^{k-1}<m+1 \leq 2^{k}$, by the induction hypothesis we have

$$
g_{m}=(-1)^{m+1} g_{2^{k}-m-1} .
$$

By (9), the left-hand side of (10) is equal to

$$
g_{2 m}=(-1)^{m(m+1) / 2} g_{m}=(-1)^{m(m+1) / 2+m+1} g_{2^{k}-m-1} .
$$

Since $k \geq 2$, the right-hand side of (10) is equal to

$$
-g_{2^{k+1}-2 m-1}=(-1)^{\left(2^{k}-m\right)\left(2^{k}-m+1\right) / 2+1} g_{2^{k}-m-1}=(-1)^{m(m-1) / 2+1} g_{2^{k}-m-1} .
$$

Hence (10) is true.

(ii) The case $n$ odd, $n=2 m+1$ : we need to prove that

$$
g_{2 m+1}=g_{2^{k+1}-2 m-2}, \quad \text { with } \quad 2^{k}<2 m+2 \leq 2^{k+1} .
$$

Since $2^{k}<2 m+2 \leq 2^{k+1}$ is equivalent to $2^{k-1}<m+1 \leq 2^{k}$, by the induction hypothesis we have

$$
g_{m}=(-1)^{m+1} g_{2^{k}-m-1} .
$$

By (9), the two sides of (11) are equal to

$$
\begin{gathered}
g_{2 m+1}=(-1)^{(m+1)(m+2) / 2} g_{m}=(-1)^{(m+1)(m+2) / 2+m+1} g_{2^{k}-m-1}, \\
g_{2^{k+1}-2 m-2}=(-1)^{\left(2^{k}-m-1\right)\left(2^{k}-m\right) / 2} g_{2^{k}-m-1}=(-1)^{m(m+1) / 2} g_{2^{k}-m-1} .
\end{gathered}
$$

Hence (11) is true. 

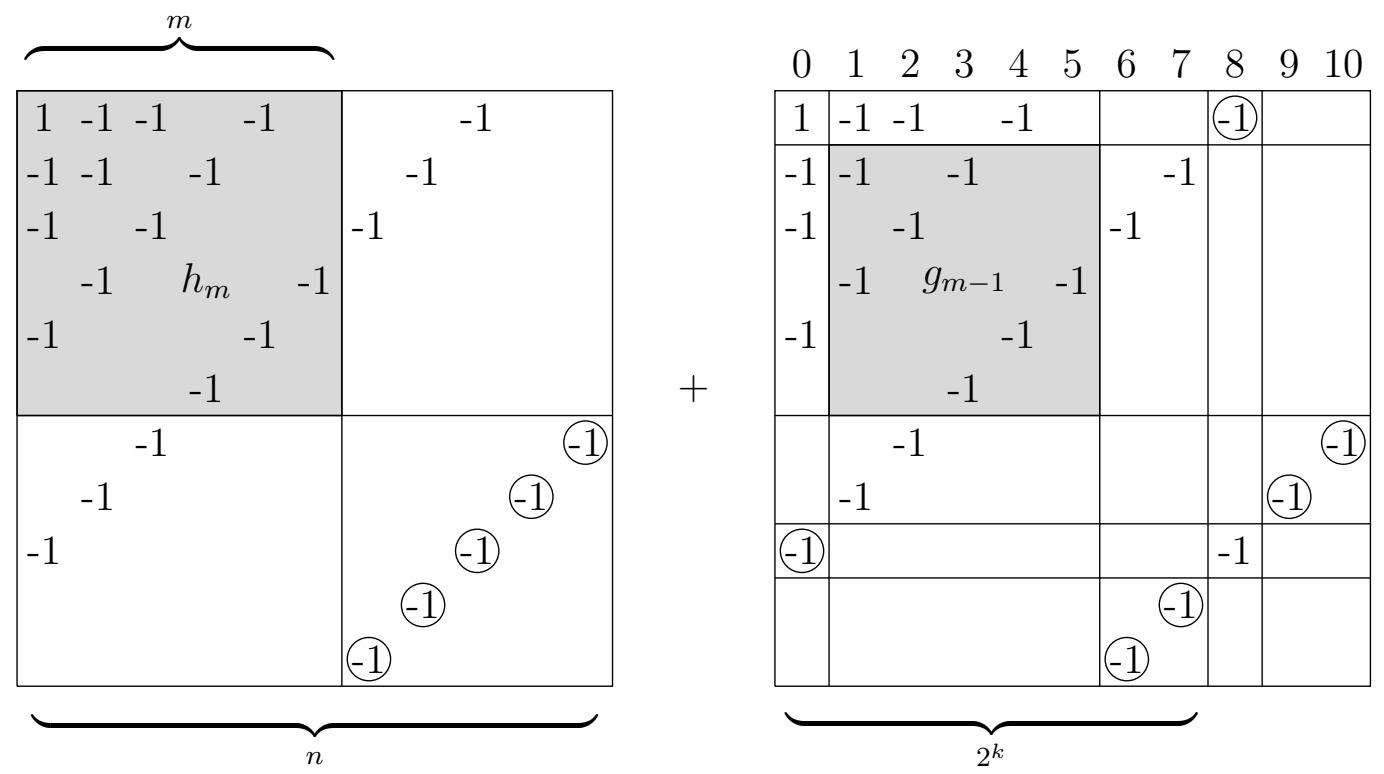

Figure 1: Hankel determinant for $n=11, k=3, m=6$

Notation 18. We recall the notation $\operatorname{sgn}(y)$ :

$$
\operatorname{sgn}(y)= \begin{cases}+1, & \text { if } y \geq 0 \\ -1, & \text { if } y<0\end{cases}
$$

Let $h_{n}=H_{n}(B(x))$.

Theorem 19. We have $h_{0}=h_{1}=1, h_{2}=-2$, and for each $n \geq 3$,

$$
h_{n}=(-1)^{n}\left(h_{m}+g_{m-1}\right),
$$

where $2^{k}<n \leq 2^{k+1}$ and $m=2^{k+1}-n+1$.

Proof. Our proof is by the fundamental properties of determinants. As illustrated in Figure 1 with the example $n=11, k=3, m=6$, we have

$$
h_{n}=(-1)^{(n-m)(n-m+1) / 2} h_{m}+(-1)^{(n-m)(n-m-1) / 2+1} g_{m-1} .
$$

Since $m=2^{k+1}-n+1$ or $n-m=2 n-2^{k+1}-1$, the above identity implies (12).

Lemma 20. For each $n \geq 1$ we have $h_{n} \neq 0$ and

$$
\operatorname{sgn}\left(h_{n}\right)=g_{n-1}
$$


Proof. We prove (13) by induction on $n$. First we check that (13) is true for $n=1,2$. Suppose that (13) is true for $1,2, \ldots, n-1$. By Theorem 19 and Lemma 17, we have

$$
h_{n}=(-1)^{n}\left(h_{m}+g_{m-1}\right)=(-1)^{n} g_{m-1}\left(\left|h_{m}\right|+1\right)=g_{n-1}\left(\left|h_{m}\right|+1\right),
$$

where $2^{k}<n \leq 2^{k+1}$ and $m=2^{k+1}-n+1$. So $h_{n} \neq 0$ and $\operatorname{sgn}\left(h_{n}\right)=g_{n-1}$.

Lemma 20 and Theorem 19 imply the following corollary about the absolute values of the Hankel determinants of $B(x)$.

Corollary 21. We have $\left|h_{0}\right|=\left|h_{1}\right|=1$ and

$$
\left|h_{n}\right|=\left|h_{2^{k+1}-n+1}\right|+1,
$$

for all $n \geq 2$, where $2^{k}<n \leq 2^{k+1}$.

Now we are ready to prove Conjectures 6, 7 and 10 of P. Barry [5].

Theorem 22 (Barry's Conjecture 6). For each $n \geq 0$, we have

$$
\left|H_{n+1}(1-x r(x))\right|=s_{n},
$$

where the sequence $\left(s_{n}\right)_{n \geq 0}$ is defined in (2).

Proof. Equivalently, it suffices to prove $h_{1}=1=s_{0}$ and

$$
\left|h_{n+1}\right|-\left|h_{n}\right|=j_{n}=(-1)^{m},
$$

where $n=2^{s}(2 m+1) \geq 1$. We consider two cases.

(i) The case $n=2^{k+1}$, i.e., $m=0$ : by (14), we have

$$
\left|h_{n+1}\right|=\left|h_{2^{k+2}-n}\right|+1=\left|h_{n}\right|+1 \text {. }
$$

(ii) The case $2^{k}<n<2^{k+1}$ or $2^{k}+1<n+1 \leq 2^{k+1}$ : by (14), we have

$$
\begin{aligned}
\left|h_{n}\right| & =\left|h_{2^{k+1}-n+1}\right|+1 ; \\
\left|h_{n+1}\right| & =\left|h_{2^{k+1}-n}\right|+1 .
\end{aligned}
$$

So

$$
\left|h_{n+1}\right|-\left|h_{n}\right|=-\left(\left|h_{n^{\prime}+1}\right|-\left|h_{n^{\prime}}\right|\right)
$$

where $n^{\prime}=2^{k+1}-n<n$. Hence we can prove (16) by induction on $n$. Since $k \geq s+1$, and

$$
n^{\prime}=2^{k+1}-n=2^{k+1}-2^{s}(2 m+1)=2^{s}\left(2\left(2^{k-s}-m-1\right)+1\right),
$$

By the induction hypothesis, we get

$$
\left|h_{n^{\prime}+1}\right|-\left|h_{n^{\prime}}\right|=(-1)^{2^{k-s}-m-1}=(-1)^{m+1},
$$

so that

$$
\left|h_{n+1}\right|-\left|h_{n}\right|=-\left(\left|h_{n^{\prime}+1}\right|-\left|h_{n^{\prime}}\right|\right)=(-1)^{m} .
$$


Barry's Conjectures 7 and 10 are consequence of the above Theorem.

Corollary 23 (Barry's Conjecture 8). The sequence

$$
u_{n}=\frac{\left|\operatorname{sgn}\left(h_{n+1}\right)-\operatorname{sgn}\left(h_{n}\right)\right|}{2} \quad(n \geq 1)
$$

is the paperfolding sequence on $\{0,1\}$, i.e., $u_{2 n}=u_{n}, u_{4 n+1}=1, u_{4 n+3}=0$.

Proof. By Lemma 20,

$$
u_{n}=\frac{\left|g_{n}-g_{n-1}\right|}{2} \quad(n \geq 1)
$$

By relation (9), we have

$$
\begin{aligned}
u_{2 n} & =\frac{1}{2}\left|(-1)^{n(n+1) / 2} g_{n}-(-1)^{n(n+1) / 2} g_{n-1}\right|=u_{n} ; \\
u_{4 n+1} & =\frac{1}{2}\left|(-1)^{(2 n+1)(2 n+2) / 2} g_{2 n}-(-1)^{2 n(2 n+1) / 2} g_{2 n}\right|=\left|g_{2 n}\right|=1 ; \\
u_{4 n+3} & =\frac{1}{2}\left|(-1)^{(2 n+2)(2 n+3) / 2} g_{2 n+1}-(-1)^{(2 n+1)(2 n+2) / 2} g_{2 n+1}\right|=0 .
\end{aligned}
$$

Barry's Conjecture 9 is true by Corollary 23 (Barry's Conjecture 8) and the fact that $|a+b|+|a-b|=2$ for $a, b \in\{-1,1\}$.

We give an algorithmic description of the sequence $H(B(x))$. For $y \in \mathbb{Z}$, we define

$$
y^{+}= \begin{cases}y+1, & \text { if } y>0 \\ y-1, & \text { if } y \leq 0\end{cases}
$$

Note that for $\left|y^{+}\right|=|y|+1$ and that $\operatorname{sgn}\left(y^{+}\right)=\operatorname{sgn}(y)$ for $y \neq 0$.

Theorem 24. The Hankel determinants $h_{n}$ of the sequence $B(x)$ are characterized by $h_{0}=$ $h_{1}=1, h_{2}=-2$ and
(a) $h_{8 n}=h_{4 n}$
(b) $h_{8 n+1}=h_{4 n+1}$
(c) $h_{8 n+2}=h_{4 n+2}$
(d) $h_{8 n+3}=-\left(h_{4 n+2}\right)^{+}$
(e) $h_{8 n+4}=-h_{4 n+2}$
(f) $h_{8 n+5}=-h_{4 n+3}$
(g) $h_{8 n+6}=\left(h_{4 n+3}\right)^{+}$
(h) $h_{8 n+7}=h_{4 n+3}$. 
Proof. The "sign" parts of (a)-(h) are easily derived from the four identities:

$$
\begin{aligned}
\operatorname{sgn}\left(h_{2 n}\right) & =(-1)^{n(n+1) / 2} \operatorname{sgn}\left(h_{n}\right), \\
\operatorname{sgn}\left(h_{2 n+1}\right) & =(-1)^{n(n+1) / 2} \operatorname{sgn}\left(h_{n+1}\right) \\
\operatorname{sgn}\left(h_{4 n+1}\right) & =(-1)^{n} \operatorname{sgn}\left(h_{2 n+1}\right)=-\operatorname{sgn}\left(h_{4 n+2}\right) \\
\operatorname{sgn}\left(h_{4 n+4}\right) & =(-1)^{n+1} \operatorname{sgn}\left(h_{2 n+2}\right)=\operatorname{sgn}\left(h_{4 n+3}\right)
\end{aligned}
$$

The first two relations are consequences of (13) and (9), and they immediately imply the other ones.

Now it suffices to prove the statement in Theorem 24 for the "absolute values" parts. We use induction on $n$, where the induction hypothesis $\mathcal{H}_{n}$ is: the relations (a) to (h) are true for all $(k, j)$ with $j \in[0,7]$ and $8 k+j \leq 8 n$. It is easy to see that $\mathcal{H}_{0}$ is true. Now suppose $\mathcal{H}_{n}$ is true and let us prove that the relations (b) to (h) hold and that $h_{8 n+8}=h_{4 n+4}$. Let $n=2^{s}(2 r+1)$. Using relations (16) and the induction hypothesis we have:

(b) Let $n=2^{s}(2 m+1)$. Then we have

$$
\left|h_{8 n+1}\right|=\left|h_{8 n}\right|+(-1)^{m}=\left|h_{4 n}\right|+(-1)^{m}=\left|h_{4 n+1}\right| .
$$

(c) $\left|h_{8 n+2}\right|=\left|h_{8 n+1}\right|+1=\left|h_{4 n+1}\right|+1=\left|h_{4 n+2}\right|$.

(d) $\left|h_{8 n+3}\right|=\left|h_{8 n+2}\right|+1=\left|h_{4 n+2}\right|+1=\left|\left(h_{4 n+2}\right)^{+}\right|$.

(e) $\left|h_{8 n+4}\right|=\left|h_{8 n+3}\right|-1=\left|\left(h_{4 n+2}\right)^{+}\right|-1=\left|h_{4 n+2}\right|$.

(f) $\left|h_{8 n+5}\right|=\left|h_{8 n+4}\right|+(-1)^{n}=\left|h_{4 n+2}\right|+(-1)^{n}=\left|h_{4 n+3}\right|$.

(g) $\left|h_{8 n+6}\right|=\left|h_{8 n+5}\right|+1=\left|h_{4 n+3}\right|+1=\left|\left(h_{4 n+3}\right)^{+}\right|$.

(h) $\left|h_{8 n+7}\right|=\left|h_{8 n+6}\right|-1=\left|\left(h_{4 n+3}\right)^{+}\right|-1=\left|h_{4 n+3}\right|$.

and finally

(a) $\left|h_{8 n+8}\right|=\left|h_{8 n+7}\right|-1=\left|h_{4 n+3}\right|-1=\left|h_{4 n+4}\right|$.

The sequences $\left(g_{n}\right)_{n \geq 0}$ and $\left(h_{n}\right)_{n \geq 0}$ are somewhat related to the (regular) paperfolding sequence, which is a 2-automatic sequence. It is thus natural to ask whether they are automatic or regular (for more about $d$-automatic and $d$-regular sequences, the reader can consult [4], in particular Chapters 5 and 16). This question will be answered in the next two theorems.

Theorem 25. The sequence $\left(g_{n}\right)_{n \geq 0}$ is 2-automatic.

Proof. To prove that the sequence $\left(g_{n}\right)_{n \geq 0}$ is 2 -automatic, we have to prove that the set of subsequences $\left\{\left(g_{2^{n}+j}, n \geq 0, j \in\left[0,2^{k-1}\right]\right\}\right.$ is finite. It suffices to prove the following relations:

$$
\begin{aligned}
& g_{4 n+1}=g_{2 n+1} \\
& g_{4 n+2}=g_{2 n} \\
& g_{4 n+3}=g_{2 n} \\
& g_{8 n}=g_{4 n} \\
& g_{16 n+4}=g_{2 n+1} \\
& g_{16 n+12}=g_{8 n+4}
\end{aligned}
$$


for all $n \geq 0$. We have seen in Equation (9) that $\left(g_{n}\right)$ satisfies

$$
g_{2 n}=(-1)^{n(n+1) / 2} g_{n} \text { and } g_{2 n+1}=(-1)^{(n+1)(n+2) / 2} g_{n}
$$

for all $n \geq 0$. Let us define $\gamma_{n}=(-1)^{n(n+1) / 2}$ and $\delta_{n}=(-1)^{(n+1)(n+2) / 2}$ so that $g_{2 n}=\gamma_{n} g_{n}$ and $g_{2 n+1}=\delta_{n} g_{n}$. It easy to see that

$$
\begin{aligned}
\gamma_{2 n} & =(-1)^{n}, \quad \gamma_{2 n+1}=\delta_{2 n}=\delta_{2 n+1}=(-1)^{n+1}, \\
\delta_{2 n} \gamma_{n} & =\delta_{n}=-\gamma_{2 n} \gamma_{n}, \quad \gamma_{2 n+1} \delta_{n}=\delta_{2 n+1} \delta_{n}=\gamma_{n} .
\end{aligned}
$$

Thus

$$
\begin{aligned}
g_{4 n+1} & =g_{2(2 n)+1}=\delta_{2 n} g_{2 n}=\delta_{2 n} \gamma_{n} g_{2 n}=\delta_{n} \gamma_{n}^{2} g_{n}=\delta_{n} g_{n}=g_{2 n+1} \\
g_{4 n+2} & =g_{2(2 n+1)}=\gamma_{2 n+1} g_{2 n+1}=\gamma_{2 n+1} \delta_{n} g_{n}=\gamma_{n} g_{n}=g_{2 n} \\
g_{4 n+3} & =g_{2(2 n+1)+1}=\delta_{2 n+1} g_{2 n+1}=\delta_{2 n+1} \delta_{n} g_{n}=\gamma_{n} g_{n}=g_{2 n} \\
g_{8 n} & =g_{2(4 n)}=\gamma_{4 n} g_{4 n}=(-1)^{2 n} g_{4 n}=g_{4 n} .
\end{aligned}
$$

Now

$$
\begin{aligned}
g_{4 n} & =g_{2(2 n)}=\gamma_{2 n} g_{2 n}=\gamma_{2 n} \gamma_{n} g_{n}=(-1)^{n} \gamma_{n} g_{n} \quad \text { which implies } \\
g_{8 n+4} & =g_{4(2 n+1)}=-\gamma_{2 n+1} g_{2 n+1}=-\gamma_{2 n+1} \delta_{n} g_{n}=-\gamma_{n} g_{n} .
\end{aligned}
$$

Hence

$$
\begin{aligned}
g_{16 n+4} & =g_{8(2 n)+4}=-\gamma_{2 n} g_{2 n}=-\gamma_{2 n} \gamma_{n} g_{n}=\delta_{n} g_{n}=g_{2 n+1} \\
g_{16 n+12} & =g_{8(2 n+1)+4}=-\gamma_{2 n+1} g_{2 n+1}=-\gamma_{2 n+1} \delta_{n} g_{n}=-\gamma_{n} g_{n}=g_{8 n+4}
\end{aligned}
$$

Now we prove that the sequence $\left(h_{n}\right)_{n \geq 0}$ is 2-regular (see [4, Chapter 16] for more on $d$-regular sequences).

Theorem 26. The sequence $\left(h_{n}\right)_{n \geq 0}$ is 2-regular.

Proof. To prove the 2-regularity of $\left(h_{n}\right)_{n \geq 0}$, we establish, using Theorem 24, the following equalities:

$$
\begin{aligned}
h_{8 n} & =h_{4 n} \\
h_{8 n+1} & =h_{4 n+1} \\
h_{8 n+2} & =h_{4 n+2} \\
h_{8 n+3} & =-h_{4 n+1}-2 h_{4 n+2} \\
h_{8 n+4} & =-h_{4 n+2} \\
h_{8 n+5} & =-h_{4 n+3} \\
h_{8 n+6} & =-h_{2 n+1}+h_{4 n+1}+h_{4 n+2}+2 h_{4 n+3} \\
h_{8 n+7} & =h_{4 n+3}
\end{aligned}
$$

for all $n \geq 0$. All these equalities but two of them have been already proved in Theorem 24 . It remains to prove that

(I) $h_{8 n+3}=-h_{4 n+1}-2 h_{4 n+2}$

(II) $h_{8 n+6}=-h_{2 n+1}+h_{4 n+1}+h_{4 n+2}+2 h_{4 n+3}$ 
for all $n \geq 0$. By (16) and Theorem 24, we have

$$
\left|h_{8 n+3}\right|+\left|h_{4 n+1}\right|=2\left|h_{4 n+2}\right| \text { and } \operatorname{sgn}\left(h_{8 n+3}\right)=-\operatorname{sgn}\left(h_{4 n+2}\right)=\operatorname{sgn}\left(h_{4 n+1}\right)
$$

This implies (I). Similary, we have

$$
\left|h_{8 n+6}\right|+\left|h_{4 n+4}\right|=2\left|h_{4 n+3}\right| \text { and } \operatorname{sgn}\left(h_{8 n+6}\right)=\operatorname{sgn}\left(h_{4 n+3}\right)=\operatorname{sgn}\left(h_{4 n+4}\right) .
$$

So

$$
h_{8 n+6}=2 h_{4 n+3}-h_{4 n+4} .
$$

On the other hand, we have

$$
\begin{aligned}
& h_{4 n+1}=h_{8 n+1}+h_{8 n+2}+h_{8 n+4} \\
& h_{4 n+3}=h_{8 n+5}+h_{8 n+6}+h_{8 n+8} .
\end{aligned}
$$

Combining the above two identities yields

$$
h_{2 n+1}=h_{4 n+1}+h_{4 n+2}+h_{4 n+4} .
$$

Identity (II) is deduced from (18) and (19).

Remark 27. The 2-automaticity of $\left(g_{n}\right)$ proved in Theorem 25 can also be proved directly from the four identities at the beginning of the proof of Theorem 24 and the fact that the sequences $(-1)^{n},(-1)^{n+1}$ and $(-1)^{n(n+1) / 2}$ are periodic.

It is also possible to deduce the 2-automaticity of $\left(g_{n-1}\right)_{n \geq 1}$ (hence of $\left.\left(g_{n}\right)_{n \geq 0}\right)$ from the relations satisfied by the $h_{n}$ 's in Theorem 24, and Lemma 20. An important caveat is that it is not true in general that the sequence of signs of a $d$-regular sequence is $d$-automatic. Here is an example concocted from [2, p. 168-169]: let $e_{0}(n)$ and $e_{1}(n)$ count, respectively, the number of 0's and the number of 1's in the binary expansion of the integer $n$. It is easy to see that $\left(e_{0}(n)\right)_{n}$ and $\left(e_{1}(n)\right)_{n}$ are 2-regular, so is $(f(n))_{n}$ defined by $f(n)=e_{0}(n)-e_{1}(n)$. It is proved in [2, p. 168-169] that $(|f(n)|)_{n}$ is not 2-regular. If $(\operatorname{sgn}(f(n)))_{n}$ were 2-automatic, hence 2-regular, the product sequence $(\operatorname{sgn}(f(n))|f(n)|)_{n}$ would be 2-regular as well. But $\operatorname{sgn}(f(n))|f(n)|=f(n)$, a contradiction.

\section{The Hankel determinants of $1+x r(x)$}

Remark 28. In this section we re-use the symbols $B(x), T(x), g_{n}, h_{n}$ with meanings different from those in the previous section. We trust there will be no confusion.

Let

$$
B(x)=1+x r(x)=1+\sum_{k \geq 0} x^{2^{k}}=1+x+x^{2}+x^{4}+x^{8}+x^{16}+\cdots
$$

The first terms of the Hankel determinants of $B(x)$ are

$$
H(B(x))=(1,1,0,-1,0,1,2,-1,0,1,2,3,-2,1,2,-1,0,1,2,3, \ldots)
$$


Consider the sequence $\left(r_{1}, r_{2}, r_{3}, \ldots\right)$ obtained from $B(x)$ by shifting two times, i.e.,

$$
T(x)=\frac{B(x)-(1+x)}{x^{2}}=\frac{r-1}{x} .
$$

It is also the shifted Rueppel sequence. Let $g_{n}=H_{n}(T(x))$. From Lemma 17 and relation (9), we have $g_{0}=g_{1}=1$, and

$$
g_{2 n}=(-1)^{n(n-1) / 2} g_{n}, \quad g_{2 n+1}=(-1)^{n(n+1) / 2} g_{n} .
$$

and

where $2^{k}<n+1 \leq 2^{k+1}$.

$$
g_{n}=(-1)^{n} g_{2^{k+1}-n-1},
$$

Let $h_{n}=H_{n}(B(x))$.

Theorem 29. We have $h_{0}=h_{1}=1$. For each $n \geq 2$, we have

$$
h_{n}=(-1)^{n-1}\left(h_{m}-g_{m-1}\right) .
$$

where $2^{k}<n \leq 2^{k+1}$ and $m=2^{k+1}-n+1$.

Proof. Our proof is by the fundamental properties of determinants. Similar to the illustrated in Figure 1 with the example $n=11, k=3, m=6$, we have

$$
h_{n}=(-1)^{(n-m)(n-m-1) / 2} h_{m}+(-1)^{(n-m-1)(n-m-2) / 2+1} g_{m-1} .
$$

Since $m=2^{k+1}-n+1$, we have $n-m=2 n-2^{k+1}-1$. The above identity implies (23).

Lemma 30. For each $n \geq 3$ we have

$$
\operatorname{sgn}\left(h_{n}\right)=-g_{n-1},
$$

with the convention that $\operatorname{sgn}(0)=+1$.

Proof. We prove (24) by induction on $n$. First we check that (24) is true for $n=3,4,5$. Suppose that (24) is true for $3,4, \ldots, n-1$. Let $2^{k}<n \leq 2^{k+1}$ and $m=2^{k+1}-n+1$. We consider three cases.

(i) The case $m=1$ : by Theorem 29 and relation (22), we have

$$
h_{n}=(-1)^{n-1}\left(h_{1}-g_{0}\right)=0 .
$$

So $\operatorname{sgn}\left(h_{n}\right)=1=-g_{n-1}$.

(ii) The case $m=2$ : we have

$$
h_{n}=(-1)^{n-1}\left(h_{2}-g_{1}\right)=(-1)^{n}=-1 .
$$

So $\operatorname{sgn}\left(h_{n}\right)=-1=-g_{n-1}$.

(iii) The case $m \geq 3$ : we have

$$
h_{n}=(-1)^{n-1}\left(h_{m}-g_{m-1}\right)=(-1)^{n} g_{m-1}\left(\left|h_{m}\right|+1\right)=-g_{n-1}\left(\left|h_{m}\right|+1\right),
$$

So $\operatorname{sgn}\left(h_{n}\right)=-g_{n-1}$. 
Lemma 30 and Theorem 29 imply the following corollary about the absolute values of the Hankel determinants of $B(x)$.

Corollary 31. We have $\left|h_{0}\right|=\left|h_{1}\right|=1,\left|h_{2}\right|=0,\left|h_{2^{k+1}}\right|=0$, and

$$
\left|h_{n}\right|=\left|h_{2^{k+1}-n+1}\right|+1 \text {, }
$$

where $2^{k}<n<2^{k+1}$.

Now we are ready to prove Conjecture 11 of P. Barry [5].

Theorem 32 (Barry's Conjecture 11). For each $n \geq 1$ we have

$$
\left|H_{n+1}(1-x r(x))\right|=s_{n}-2,
$$

where the sequence $\left(s_{n}\right)_{n \geq 0}$ is defined in (2).

Proof. Equivalently, it suffices to prove $h_{2}=0=s_{1}-2$ and

$$
\left|h_{n+1}\right|-\left|h_{n}\right|=j_{n}=(-1)^{m},
$$

where $n=2^{s}(2 m+1) \geq 2$. We consider three cases.

(i) The case $n=2^{k+1}$, i.e., $m=0$ : by (25), we have

$$
\left|h_{n+1}\right|=\left|h_{2^{k+2}-n}\right|+1=\left|h_{n}\right|+1 .
$$

(ii) The case $n=2^{k+1}-1$, i.e., $m=2^{k}-1$ : by (25), we have

$$
\begin{aligned}
\left|h_{n}\right| & =\left|h_{2^{k+1}-n+1}\right|+1=1 ; \\
\left|h_{n+1}\right| & =0 .
\end{aligned}
$$

So

$$
\left|h_{n+1}\right|-\left|h_{n}\right|=-1=(-1)^{m} .
$$

(iii) The case $2^{k}<n<2^{k+1}-1$ or $2^{k}+1<n+1<2^{k+1}$ : by (25), we have

$$
\begin{aligned}
\left|h_{n}\right| & =\left|h_{2^{k+1}-n+1}\right|+1 ; \\
\left|h_{n+1}\right| & =\left|h_{2^{k+1}-n}\right|+1 .
\end{aligned}
$$

So

$$
\left|h_{n+1}\right|-\left|h_{n}\right|=-\left(\left|h_{n^{\prime}+1}\right|-\left|h_{n^{\prime}}\right|\right) \text {, }
$$

where $n^{\prime}=2^{k+1}-n<n$. Hence we can prove (27) by induction on $n$. Since $k \geq s+1$, and

$$
n^{\prime}=2^{k+1}-n=2^{k+1}-2^{s}(2 m+1)=2^{s}\left(2\left(2^{k-s}-m-1\right)+1\right),
$$

By the induction hypothesis, we get

$$
\left|h_{n^{\prime}+1}\right|-\left|h_{n^{\prime}}\right|=(-1)^{2^{k-s}-m-1}=(-1)^{m+1},
$$

so that

$$
\left|h_{n+1}\right|-\left|h_{n}\right|=-\left(\left|h_{n^{\prime}+1}\right|-\left|h_{n^{\prime}}\right|\right)=(-1)^{m} .
$$


We give an algorithmic description of the sequence $H(B(x))$. Recall that $y^{+}$is defined by (17) for each $y \in \mathbb{Z}$.

Theorem 33. The Hankel determinants $h_{n}$ of the sequence $B(x)$ are characterized by $h_{0}=$ $h_{1}=1, h_{2}=0$ and
(a) $h_{8 n}=h_{4 n}$
(b) $h_{8 n+1}=h_{4 n+1}$
(c) $h_{8 n+2}=h_{4 n+2}$
(d) $h_{8 n+3}=\left(h_{4 n+2}\right)^{+}$
(e) $h_{8 n+4}=-h_{4 n+2}$
(f) $h_{8 n+5}=-h_{4 n+3}$
(g) $h_{8 n+6}=-\left(h_{4 n+3}\right)^{+}$
(h) $h_{8 n+7}=h_{4 n+3}$.

Proof. The "sign" parts of (a)-(h) are easily derived from the four identities:

$$
\begin{aligned}
\operatorname{sgn}\left(h_{2 n}\right) & =(-1)^{n(n-1) / 2} \operatorname{sgn}\left(h_{n}\right), \\
\operatorname{sgn}\left(h_{2 n+1}\right) & =(-1)^{n(n-1) / 2} \operatorname{sgn}\left(h_{n+1}\right) \\
\operatorname{sgn}\left(h_{4 n+1}\right) & =(-1)^{n} \operatorname{sgn}\left(h_{2 n+1}\right)=\operatorname{sgn}\left(h_{4 n+2}\right) \\
\operatorname{sgn}\left(h_{4 n+4}\right) & =(-1)^{n+1} \operatorname{sgn}\left(h_{2 n+2}\right)=-\operatorname{sgn}\left(h_{4 n+3}\right)
\end{aligned}
$$

(the first two relations are consequences of (24) and (21), and they immediately imply the other ones). Comparing Theorems 22 and 32 we have

$$
\left|H_{n}(1+x r(x))\right|=\left|H_{n}(1-x r(x))\right|-2,
$$

for $n \geq 2$. The "absolute values" parts are treated as in the proof of Theorem 24, using the above relation.

Theorem 34. The sequence $\left(g_{n}\right)_{n \geq 0}$ is 2-automatic.

Proof. To prove that the sequence $\left(g_{n}\right)_{n \geq 0}$ is 2-automatic, we have to prove that the set of subsequences $\left\{\left(g_{2^{n}+j}, n \geq 0, j \in\left[0,2^{k-1}\right]\right\}\right.$ is finite. It suffices to prove the following relations:

$$
\begin{aligned}
& g_{4 n}=g_{2 n+1} \\
& g_{4 n+1}=g_{2 n+1} \\
& g_{4 n+2}=g_{2 n} \\
& g_{8 n+7}=g_{4 n+3} \\
& g_{16 n+3}=g_{8 n+3} \\
& g_{16 n+11}=g_{2 n}
\end{aligned}
$$

for all $n \geq 0$. This can be done from Identity (21) by using the same method in Theorem 25 .

Now we prove that the sequence $\left(h_{n}\right)_{n \geq 0}$ is 2-regular (see [4, Chapter 16] for more on $d$-regular sequences).

Theorem 35. The sequence $\left(h_{n}\right)_{n \geq 0}$ is 2-regular. 
Proof. To prove the 2-regularity of $\left(h_{n}\right)_{n \geq 0}$, we establish, using Theorem 24, the following equalities:

$$
\begin{aligned}
h_{8 n} & =h_{4 n} \\
h_{8 n+1} & =h_{4 n+1} \\
h_{8 n+2} & =h_{4 n+2} \\
h_{8 n+3} & =-h_{4 n+1}+2 h_{4 n+2} \\
h_{8 n+4} & =-h_{4 n+2} \\
h_{8 n+5} & =-h_{4 n+3} \\
h_{8 n+6} & =h_{2 n+1}-h_{4 n+1}+h_{4 n+2}-2 h_{4 n+3} \\
h_{8 n+7} & =h_{4 n+3}
\end{aligned}
$$

for all $n \geq 0$. This can be done by using the same method as in Theorem 26 .

\section{The Hankel determinants of $r(x) /(r(x)-x)$}

Let us recall the following useful result [11, Lemma 2.2]

Lemma 36. Let $k$ be a nonnegative integer and let $F(x), G(x)$ be two power series satisfying

$$
F(x)=\frac{x^{k}}{1+u(x) x-x^{k+2} G(x)},
$$

where $u(x)$ is a polynomial of degree less than or equal to $k$. Then we have

$$
H_{n}(F)=(-1)^{k(k+1) / 2} H_{n-k-1}(G) .
$$

Let

$$
B_{0}(x)=\frac{r(x)}{r(x)-x}=1+x-x^{4}+x^{7}-x^{8}-x^{10}+2 x^{11}+\cdots
$$

The first terms of the Hankel determinants of $B_{0}(x)$ are

$$
H\left(B_{0}(x)\right)=(1,1,-1,1,1,-1,1,1,1,-1,1,-1,-1,-1,1,1,1, \ldots)
$$

Theorem 37 (Barry's Conjecture 16).

$$
H_{n}\left(\frac{r(x)}{r(x)-x}\right)=\operatorname{sgn}\left(H_{n}(1-x r(x))\right) .
$$

Proof. We have

$$
B_{0}(x)=\frac{1}{1-x-x^{2} B_{1}(x)},
$$

where

$$
-B_{1}(x)=\frac{r-1}{x r}=\frac{1}{1+x-x^{2} U(x)},
$$


and where

$$
U(x)=\frac{r-1-x}{(r-1) x^{2}}
$$

Also, Let

$$
G(x)=\frac{1-r(x)}{x}
$$

Then

$$
-G(x)=\frac{1}{1-x^{2} U(x)} .
$$

By Lemma 36 we have, successively,

$$
\begin{aligned}
H_{n}\left(B_{0}\right) & =H_{n-1}\left(B_{1}\right), \\
(-1)^{n} H_{n}\left(B_{1}\right) & =H_{n-1}(U), \\
(-1)^{n} H_{n}(G) & =H_{n-1}(U) .
\end{aligned}
$$

So by Lemma 20, we derive the equality

$$
H_{n}\left(B_{0}\right)=\operatorname{sgn}\left(H_{n}(1-x r)\right) .
$$

\section{Acknowledgments}

The first author warmly thanks F. Durand for discussions about Cobham's theorem and its generalizations.

\section{References}

[1] J.-P. Allouche, B. Cloitre, and V. Shevelev. Beyond odious and evil. Aequationes Math. 90 (2016), 341-353.

[2] J.-P. Allouche and J. Shallit. The ring of $k$-regular sequences. Theoret. Comput. Sci., 98 (1992) 163-197.

[3] J.-P. Allouche and J. Shallit. The ubiquitous Prouhet-Thue-Morse sequence. In Sequences and Their Applications (Singapore, 1998), Springer Ser. Discrete Math. Theor. Comput. Sci., Springer, London, 1999, pp. 1-16.

[4] J.-P. Allouche and J. Shallit. Automatic Sequences. Theory, Applications, Generalizations. Cambridge University Press, Cambridge, 2003.

[5] P. Barry. Some observations on the Rueppel sequence and associated Hankel determinants. Preprint, 2020, https://arxiv.org/abs/2005.04066. 
[6] J. Cigler. Some observations about determinants which are connected with Catalan numbers and related topics. Preprint, 2019, https://arxiv.org/abs/1902.10468.

[7] E. Cateland. Suites digitales et suites k-régulières. Thèse, Université Bordeaux 1, 1992. Available at https://tel.archives-ouvertes.fr/tel-00845511.

[8] M. Dekking. Morphic words, Beatty sequences and integer images of the Fibonacci language. Theoret. Comput. Sci. 809 (2020), 407-417.

[9] E. Deutsch and B. E. Sagan. Congruences for Catalan and Motzkin numbers and related sequences. J. Number Theory 117 (2006), 191-215.

[10] F. Durand. Cobham's theorem for substitutions. J. Eur. Math. Soc. 13 (2011), 17991814.

[11] G.-N. Han. Hankel continued fraction and its applications. Adv. Math. 303 (2016), $295-321$.

[12] J. Lambek and L. Moser. Inverse and complementary sequences of natural numbers. Amer. Math. Monthly 61 (1954), 454-458.

[13] M. Mendès France. Principe de la symétrie perturbée. In Sémin. Delange-Pisot-Poitou, Paris 1979-80. Reprinted in Prog. Math. 12, Birkhäuser, 1981, pp. 77-98.

[14] M. Mendès France. Principe de la symétrie perturbée, papiers pliés et dimension des courbes. In Séminaire de Philosophie et Mathématiques, 1981, fascicule 7, pp. 1-11.

[15] N. J. A. Sloane et al. On-Line Encyclopedia of Integer Sequences. Available at https: //oeis.org.

[16] J. Shallit. The mathematics of Per Nørgård's rhythmic infinity system. Fibonacci Quart. 43 (2005), 262-268. 